\title{
Caracterización fisicoquímica y microbiológica de aguas mieles del beneficio del café
}

\section{Physicochemical and microbiological characterization of wastewater from coffee processing}

\author{
Caracterização físico-química e microbiológica de \\ águas residuais a partir do benefício do café
}

\author{
Laura Sofía Torres-Valenzuela*; Alejandra Sanín-Villarrea; Andrea Arango-Ramírez; \\ Johanna Andrea Serna-Jiménez \\ Facultad de Ingenierías. Universidad La Gran Colombia, Campus Ciudadela del Saber La Santa María, Km 7 vía \\ Armenia - La Tebaida, Armenia, Colombia \\ torresvallaura@miugca.edu.co
}

Fecha recepción: 15 de octubre de 2019 Fecha aceptación: 13 de diciembre de 2019

\begin{abstract}
Resumen
En el procesamiento del café, sólo se aprovecha el $5 \%$ del producto fresco y se generan residuos como aguas mieles (AM), que pueden llegar hasta $40 \mathrm{~L} / \mathrm{kg}$ de café pergamino seco y al ser vertidas pueden provocar contaminación de alto impacto ambiental. Consecuentemente, el objetivo fue caracterizar fisicoquímica y microbiológicamente dos muestras de AM (M1, M2) con el fin de evaluar el potencial en la extracción de biocomponentes. Los parámetros medidos a las dos muestras de agua fueron Demanda Química de Oxígeno (DQO), nitrógeno, amonio, cromo, oxígeno disuelto (OD), pH, conductividad, acidez volátil, fósforo, cloruros, sólidos, color y, adicionalmente se cuantificaron mesófilos, coliformes totales y estafilococos. Se identificaron diferencias significativas entre las muestras y un efecto del procesamiento del café, sobre las características evaluadas. M2 mostró mayor concentración de OD, conductividad y cambio de color respecto al control, mientras que M1 tuvo mayor concentración para los demás parámetros evaluados. Cromo y amonio estuvieron por debajo del límite de detección de la prueba empleada, por el contrario, la DQO fue superior a lo reglamentado para aguas domésticas. En el análisis microbiológico, se encontraron mesófilos en ambas muestras, y M2 presentó coliformes y estafilococos. Con lo anterior se evidencia que el método de procesamiento de café afecta los parámetros de calidad de las aguas residuales y por ende se deben implementar metodologías de tratamiento y/o aprovechamiento acordes a las características intrínsecas de cada proceso.
\end{abstract}

Palabras clave: Aguas Residuales; Biocomponentes; Café; Contaminación; Microorganismos.

\begin{abstract}
In the coffee processing, only $5 \%$ of the fresh product is used and waste is generated as wastewater (WW), which can reach up to $40 \mathrm{~L} / \mathrm{kg}$ of dry parchment coffee and when spilled can cause pollution of high environmental impact. Consequently, the objective was to characterize physicochemical and microbiologically two WW samples (M1, M2) in order to evaluate the potential in the extraction of biocomponents. The parameters measured to the two water samples were Chemical Oxygen Demand (COD), nitrogen, ammonium, chromium, dissolved oxygen (DO), $\mathrm{pH}$, conductivity, volatile acidity, phosphorus, chlorides, solids, color and, in addition, mesophiles, total coliforms and staphylococci were also quantified. Significant differences between the samples and an effect of coffee processing on the characteristics evaluated were identified. M2 showed a higher concentration of OD, conductivity and color change with respect to the control, while M1 had a higher concentration for the other parameters.


Chromium and ammonium were below the detection limit of the test used, on the contrary, the COD was higher than what was regulated for domestic waters. In the microbiological analysis, mesophiles were found in both samples, and M2 presented coliforms and staphylococci. With the above it is evident that the coffee processing method affects the parameters of wastewater quality and therefore treatment and/ or exploitation methodologies must be implemented according to the intrinsic characteristics of each process.

Keywords: Wastewater; Biocomponents; Coffee; Pollution; Microorganisms.

\section{Resumo}

No processamento de café, apenas $5 \%$ do produto fresco é utilizado e os resíduos são gerados como águas residuais (AR), que pode chegar a $40 \mathrm{~L} / \mathrm{kg}$ de café em pergaminho seco e quando derramado pode causar poluição de alto impacto ambiental. Consequentemente, o objetivo foi caracterizar fisicoquímica e microbiologicamente duas amostras de AR (M1, M2), a fim de avaliar o potencial na extração de biocomponentes. Os parâmetros medidos para as duas amostras de água foram: Demanda Química de Oxigênio (DQO), nitrogênio, amônio, cromo, oxigênio dissolvido (DO), pH, condutividade, acidez volátil, fósforo, cloretos, sólidos, cor e, além disso, mesófilos, coliformes totais e estafilococos também foram quantificados. Diferenças significativas entre as amostras e um efeito do processamento do café nas características avaliadas foram identificadas. M2 apresentou maior concentração de OD, condutividade e mudança de cor em relação ao controle, enquanto M1 apresentou maior concentração para os demais parâmetros. O cromo e o amônio estavam abaixo do limite de detecção do teste utilizado; pelo contrário, o DQO foi superior ao regulamentado para as águas domésticas. Na análise microbiológica, mesófilos foram encontrados em ambas as amostras, e M2 apresentou coliformes e estafilococos. Com o exposto, é evidente que o método de processamento do café afeta os parâmetros da qualidade das águas residuais e, portanto, as metodologias de tratamento e/ou exploração devem ser implementadas de acordo com as características intrínsecas de cada processo.

Palavras-chave: Águas Residuais; Biocomponentes; Café; Poluição; Microorganismos.

\section{Introducción}

El café es el producto del tipo "commodity" más demandado en el mundo, seguido de los aceites comestibles y el té. Aproximadamente el $30 \%$ de la población mundial consume una vez al día una taza de café [1]. En la última década, Colombia ha incrementado la producción de café en un $30 \%$ debido a los cambios en el sistema de producción convencional [2], catalogando el grano como un producto agrícola de gran importancia económica y social, incluso, las fincas cafeteras abarcan el $66 \%$ del área total cultivada [3]. Esta actividad económica ha sido la responsable de fomentar de manera privada el progreso en las regiones cafeteras, y del mismo modo, las ganancias han sido factor fundamental para familias campesinas, las cuales han logrado una estabilidad e independencia monetaria [4].

Al igual que en otras industrias, la transformación del fruto del café genera residuos orgánicos, en este caso, solo se aprovecha el $5 \%$ del peso del fruto fresco para la preparación de la infusión, y el $95 \%$ restante se consideran subproductos, los cuales tienen diferente composición química [5]. Estos subproductos tienen escaso o nulo aprovechamiento, por lo cual se limita su potencial para la obtención de nutrientes valiosos para la industria [6].

Para la obtención de una taza de café por beneficio húmedo se requieren 10 etapas: cultivo, cosecha y procesamiento de los frutos, secado, trilla, comercialización, catación, tostado, molienda y preparación de la bebida [7]. Uno de los mayores subproductos en este proceso, específicamente en las etapas de despulpado y lavado, son aguas residuales que en muchas ocasiones son vertidas a ríos u otras aguas superficiales [8] y generan problemas de contaminación de alto impacto ambiental, debido a la carga de contaminantes orgánicos que son nocivos para los afluentes hídricos, la salud humana, la flora y fauna acuática [9].

Estas aguas resultantes se denominan aguas mieles, dado que tienen en su composición diferentes tipos de azúcares, adicionalmente tienen un $\mathrm{pH}$ ácido $(4-4,5)$ y una alta cantidad de materia orgánica. Además, en su composición se 
encuentran compuestos bioactivos que podrían ser utilizados en la industria alimentaria, farmacéutica y cosmética, como cafeína, ácido acético, propiónico, valérico, fenoles, entre otros [10].

Para mitigar el problema de contaminación de estos subproductos, existen diversas técnicas como la biorremediación por fermentación en estado sólido (SSF) [11] y la fermentación sumergida, estas técnicas generan disminución de la carga tóxica del agua, pero implican alto costo e infraestructura moderna para su implementación y personal experimentado para su manejo [12]. Por lo tanto, es necesario encontrar técnicas más eficientes y económicas las cuales minimicen el impacto ambiental y reduzcan la cantidad de agua con carga contaminante vertida en las fuentes hídricas. Una alternativa es evaluar metodologías que permitan hacer tratamiento de las aguas y extracción de compuestos químicos de interés, con lo cual se obtendrían compuestos con valor en la industria.

Previo a diseñar y/o evaluar un proceso para el tratamiento de estas aguas residuales, es importante conocer su composición como punto de partida para el desarrollo de las tecnologías de tratamiento. Esta composición química puede verse afectada por diversos factores, como son la variedad de café, condiciones agronómicas, tipo de procesamiento. Por lo anterior, en este trabajo se realizó una caracterización fisicoquímica y microbiológica de dos muestras de agua provenientes de Circasia (Quindio, Colombia). con diferentes métodos de fermentación con el fin de conocer su composición y establecer posibles métodos de tratamiento y/o aprovechamiento.

\section{Metodología}

Se trabajó con dos tipos de aguas residuales de la región andina (Circasia, Colombia), obtenidas directamente de las fincas, con dos métodos de procesamiento:

Muestra 1: Municipio Circasia-Quindío, Variedad Castillo naranjal, Beneficio: 24 horas de fermentación anaeróbica, procesamiento vía húmeda.

Muestra 2: Municipio Calarcá-Quindío, Variedad Castillo, Beneficio: 14 horas de fermentación, procesamiento vía húmeda.

Las muestras de agua residual fueron almacenadas inmediatamente a $-14^{\circ} \mathrm{C}$. Previo a la medición de las propiedades químicas, estas fueron descongeladas a una temperatura de $4^{\circ} \mathrm{C}$.

\section{Caracterización fisicoquímica de las aguas mieles del café}

Se realizó la medición de la demanda química de oxígeno (DQO), nitrógeno, amonio y cromo empleando kit analíticos, como se detallan a continuación: DQO kit NANOCOLOR® test 0-29, nitrógeno kit NANOCOLOR® test $0-88$, amonio kit VISOCOLOR® ECO test 5-08 y cromo kit VISOCOLOR® ECO test 5-20. El oxígeno disuelto se obtuvo mediante el equipo portátil medidor de oxígeno disuelto MW600 DO Meter (Milwaukee, Hungría). Adicionalmente se midió el $\mathrm{pH}$ y la conductividad empleando un potenciómetro (Jenway, Inglaterra) bajo la metodología de APHAAWWA-WPCF y para el color se usó el colorímetro Minolta (color reader CR10 Minolta, Japón).

Para la determinación del color se tomaron las muestras de aguas mieles y agua del acueducto, teniendo un total de tres muestras, esta última con el fin de comparar el cambio total de color en el agua miel generada por el proceso de transformación del café con respecto al agua de acueducto rural $(\Delta E)$. Se midieron los parámetros $L^{*} a^{*} b$, donde $(L)$ es la luminosidad o relación entre blanco y negro, $a$ la relación rojo $(a+)$ y verde $(a-)$ y $b$ la relación amarillo $(a+)$ y azul $(a-)$. Estos tres parámetros fueron medidos empleando el colorímetro con el iluminante de 65. El cambio total de color se calculó utilizando la ecuación 1.

$$
\Delta E=\Delta L+\Delta a+\Delta b
$$

La ecuación 1 se empleó haciendo una comparación entre el resultado $L^{*} a * b$ de $M 1$ y agua de acueducto y el resultado $L^{*} a * b$ de $M 2$ y agua de acueducto.

La determinación de acidez volátil se realizó siguiendo el método oficial AOAC 945.08, la determinación de fósforo siguiendo el método oficial AOAC 995.11, la determinación de los sólidos totales se realizó por el método oficial AOAC 920.151, para cuantificar los sólidos minerales se empleó el protocolo oficial AOAC 940.26. Los sólidos volátiles se calcularon como la diferencia de los sólidos totales y los sólidos minerales y finalmente, la determinación de cloruros se realizó mediante el método oficial AOAC 915.01 [13].

\section{Caracterización microbiológica}

Se cuantificaron mesófilos aerobios y anaerobios, coliformes totales y estafilococo por los métodos normalizados para el análisis de aguas potables y residuales de la APHA-AWWA-WPCF [14]. 


\section{Diseño experimental y análisis estadístico}

Se empleó un diseño unifactorial con dos niveles (método de beneficio, M1 y M2). Los resultados fueron analizados mediante análisis de varianza a un nivel de significancia del $95 \%$ empleando el software Minitab v16.

\section{Resultados y discusión}

Los parámetros de calidad evaluados en las dos muestras de aguas residuales se presentan en la tabla 1. Los resultados evidencian un $\mathrm{pH}$ ácido y una compleja composición química, asociada a la materia orgánica y sólidos disueltos en ambas muestras, por lo cual pueden generar efectos negativos. El alto contenido de sólidos genera colores oscuros en el agua, adicionalmente contienen cafeína, azúcares, proteínas y compuestos fenólicos [15-18], por lo cual son susceptibles a la fermentación y a la generación de olores ofensivos [16]. Cuando los residuos de café son vertidos a cuerpos hídricos se agota el oxígeno del agua, lo cual genera asfixia acuática; adicionalmente la descarga de nutrientes puede generar eutrofización [19]. Además, el uso de las aguas residuales para riego puede afectar negativamente al crecimiento de las especies vegetales. Estudios previos han demostrado la fitotoxicidad y citotoxicidad de las aguas residuales de la industria del café y la inhibición total de la germinación en lechugas [16].

Tabla 1. Resultados de pruebas fisicoquímicas de aguas residuales del café.

\begin{tabular}{|c|c|c|}
\hline Característica & Fermentación 24 horas (M1) & Fermentación 14 horas (M2) \\
\hline $\mathrm{pH}$ & $4,025 \pm 0,035^{a}$ & $3,995 \pm 0,021^{a}$ \\
\hline Conductividad ( $\mu \mathrm{S} / \mathrm{cm})$ & $3,035 \pm 0,035^{a}$ & $1990,00 \pm 0,00^{b}$ \\
\hline Oxígeno disuelto (mg/L) & $1,25 \pm 0,070$ a & $3,05 \pm 0,070^{b}$ \\
\hline Cromo (mg/L) & \multicolumn{2}{|c|}{$<0,02$} \\
\hline Amonio (mg/L) & \multicolumn{2}{|c|}{$<0,1$} \\
\hline Nitrógeno total (mg/L) & 135 & 65,00 \\
\hline $\mathrm{DQO}(\mathrm{mg} / \mathrm{L})$ & \multicolumn{2}{|c|}{$>1500$} \\
\hline Sólidos volátiles (g/L) & $26,41 \pm 0,028^{a}$ & $9,925 \pm 0,063^{b}$ \\
\hline Sólidos minerales (g/L) & $3,29 \pm 0,070$ & $1,015 \pm 0,120^{b}$ \\
\hline Sólidos totales (g/L) & $29,705 \pm 0,091^{a}$ & $10,945 \pm 0,063^{b}$ \\
\hline Acidez volátil (mg ácido acético/L) & 90 & 60,00 \\
\hline Cloruros (mg/L) & $20 \pm 1,2^{a}$ & $18,00 \pm 0,99 a$ \\
\hline Fósforo soluble (mg/L) & $15,157 \pm 0,88^{a}$ & $7,557 \pm 1,31^{b}$ \\
\hline Color $(\Delta E)$ & 8,11 & 11,48 \\
\hline
\end{tabular}

Letras iguales indican que no hay diferencias estadísticamente significativas a un nivel de confianza del $95 \%$.

Dentro de los parámetros medidos, M2 mostró mayor concentración de oxígeno disuelto, una mayor conductividad y un mayor cambio de las coordenadas de color $L^{*} a * b(\Delta E)$ respecto a la muestra control (agua de acueducto), de esta manera, M2 presentó un color más oscuro dentro del chroma evaluado; mientras que, M1 mostró mayor concentración para el resto de las características.
Con respecto a cromo y amonio, los contenidos de las muestras estuvieron por debajo de las cantidades detectadas por el equipo de medición. Mientras que la DQO estuvo en una concentración superior a la detectada por el Kit utilizado.

En este trabajo se estudiaron las diferencias en el proceso de fermentación en aguas residuales obtenidas por vía húmeda, debido a que en esta etapa de la transformación primaria las 
enzimas naturales de las levaduras y bacterias presentes en el mucílago generan una serie de reacciones bioquímicas; la oxidación parcial de los azúcares, genera energía (ATP), alcoholes, ácidos, aldehídos, cetonas, ésteres y dióxido de carbono, generando un cambio en el color, acidez, $\mathrm{pH}$, olor y composición química del grano, que están directamente relacionados con el tiempo de duración de la etapa fermentativa [20].

Los resultados entre el pH (M1) y (M2), no tienen un efecto estadísticamente significativo (Valor-P $>0,05)$ por lo que esta variable no se ve afectada por el tipo de beneficio.

Los resultados de conductividad, oxígeno disuelto, sólidos volátiles, minerales y totales tuvieron resultados estadísticamente significativos (Valor-P $<0,05$ ), lo cual puede estar influenciado por el tiempo de fermentación de cada una de estas muestras, del mismo modo la concentración de oxígeno disuelto y todos los sólidos está directamente relacionado con el volumen de café y la cantidad de agua que se ha utilizado para el proceso.

En la tabla 2 se observa la relación de algunos parámetros evaluados comparados con los mencionados en la Resolución 631 de 2015, la cual establece los límites máximos permisibles en los vertimientos puntuales a cuerpos de aguas superficiales y a los sistemas de alcantarillado público [21].

Tabla 2. Parámetros y los valores máximos permisibles en los vertimientos puntuales a cuerpos de aguas superficiales y a los sistemas de alcantarillado público [21].

\begin{tabular}{|c|c|c|c|}
\hline \multirow[t]{2}{*}{ Parámetro } & \multirow[t]{2}{*}{ Unidades } & \multicolumn{2}{|c|}{$\begin{array}{l}\text { Beneficio de café (clasificación de la federación nacional de } \\
\text { cafeteros-FNC/Cenicafé) }\end{array}$} \\
\hline & & Proceso natural o ecológico & Proceso tradicional (Suave lavado) \\
\hline $\mathrm{pH}$ & $\begin{array}{l}\text { Unidades de } \\
\mathrm{pH}\end{array}$ & 5,00 a 9,00 & 5,00 a 9,00 \\
\hline $\begin{array}{l}\text { Demanda Química } \\
\text { de Oxígeno (DQO) }\end{array}$ & $\mathrm{mg} / \mathrm{L} \mathrm{O}_{2}$ & 3000 & 650 \\
\hline $\begin{array}{c}\text { Sólidos } \\
\text { suspendidos (SST) }\end{array}$ & $\mathrm{mg} / \mathrm{L}$ & 800 & 400 \\
\hline Fósforo Total (P) & $\mathrm{mg} / \mathrm{L}$ & No determinado & No determinado \\
\hline Nitrógeno Total (N) & $\mathrm{mg} / \mathrm{L}$ & No determinado & No determinado \\
\hline
\end{tabular}

Los valores de $\mathrm{pH}$ obtenidos en $\mathrm{M} 1$ y $\mathrm{M} 2$ están por debajo a los de la normatividad, esto se puede deber a la presencia de ácidos orgánicos en el café, así como procesos de fermentación que pueden generar la producción de ácidos y ocasionar la disminución del $\mathrm{pH}$ [22]. Autores como Fia et al. [23] encuentran un $\mathrm{pH}$ de $3,44( \pm 0,16)$, Alvarez et al. [24] registran un $\mathrm{pH}$ de 3,8 y Rodríguez et al. [25] y Olvera y Gutiérrez [26] encontraron valores de 4,6, teniendo una característica ácida en todos los casos, incluyendo el de la presente investigación. En cuanto a aguas residuales domésticas, Vásquez Sarria et al. [27] encontraron que oscila entre 6,1 y 7,7 siendo mayor $(3,98-4,05)$ al obtenido en esta investigación.

La medición de la DQO realizada a las muestras $M 1$ y M2 dió por encima de $1500 \mathrm{mg} / \mathrm{L}$, valor superior al límite máximo declarado por el Ministerio de Ambiente. Estos valores son congruentes con investigaciones reportadas donde se obtienen valores de DQO superiores a $1500 \mathrm{mg} / \mathrm{L}$, Olvera y Gutiérrez [26] obtuvieron una DQO de 8936,84 $\mathrm{mg} / \mathrm{L}$, el programa de investigación científica de Cenicafé obtuvo una DQO de $27400 \mathrm{mg} / \mathrm{L}$ [28] y Rodríguez et al. [25] una de 2904,72 mg/L. En comparación con la DQO de las aguas residuales domésticas, oscila entre 250 y $1400 \mathrm{mg} / \mathrm{L}$ [29], mientras que el resultado de esta investigación mostró que la DQO es mayor a $1500 \mathrm{mg} / \mathrm{L}$. Las diferencias en estos valores se asocian a la variedad de café, condiciones de manejo del cultivo y método de procesamiento primario.

La cantidad de nitrógeno y fósforo fue mayor en $\mathrm{M} 1$; el contenido de estos compuestos en aguas residuales se relaciona con la composición de la pulpa del grano [9], los fertilizantes utilizados en el cultivoyel compuesto residual presenteen las aguas utilizadas para el beneficio [30]. Tanto el fósforo como el nitrógeno son elementos fundamentales para el crecimiento biológico, esto significa que 
una alta concentración puede desencadenar el desarrollo de múltiples microorganismos, que disminuyen el oxígeno disuelto en el agua [31], desestabilizando el ecosistema, inclusive en muy bajas concentraciones genera un peligro potencial para el medio ambiente [32].

Los sólidos minerales reportados por Molina Guardado et al. [30] (3970 mg/L) indican ser superiores a los obtenidos en M1 y M2, valores altos de sólidos disueltos indican baja potabilidad de agua, adicionalmente, puede desencadenar reacciones fisiológicas perjudiciales para el consumidor [33]. La concentración de sólidos totales, sólidos minerales y sólidos volátiles para las aguas residuales domésticas se encuentra entre 476 - 1197 mg/L, 276 - 850 mg/L y 194 - 358 $\mathrm{mg} / \mathrm{L}$, respectivamente [34], mientras que en el caso de las aguas residuales del beneficio del café, están entre 10991,34 - 29642,73 mg/L, 1106,60 $3249,99 \mathrm{mg} / \mathrm{L}$ y $9884,74-26392,74 \mathrm{mg} / \mathrm{L}$. Esta variable se asocia con la turbidez del agua, que puede tener impacto negativo en el ecosistema, teniendo en cuenta que se reduce la penetración de la luz, por lo cual la tasa de fotosíntesis de las especies vegetales presentes baja y con ello la disponibilidad de alimentos en el ecosistema acuático disminuye también [34].

EI OD puede ser un indicador del grado de contaminación del agua y la posibilidad de soportar vida vegetal y animal; a mayor concentración de OD mejor calidad de agua. La mayoría de los ríos y riachuelos requieren un mínimo de 5-6 mg/L [30], por lo tanto, el resultado de estas muestras indica una mala calidad de agua para albergar vida.

En cuanto a la presencia de cromo y amonio, las muestras no resultaron tener una concentración significativa por lo que no se comparan con otras investigaciones, pero es un resultado positivo ya que altas concentraciones de estos químicos generan efectos nocivos para el medio ambiente, incluyendo el ser humano.

Por otra parte, en cuánto al color, M2 presentó un mayor cambio de coordenadas de color respecto al agua de acueducto, es decir que su color fue más intenso que el de la M1, Molina Guardado et al. [30] afirman que el color en el agua es producido por los minerales disueltos, colorantes o ácidos húmicos de las plantas, así como por la descomposición de la lignina que produce compuestos coloreados de taninos y acido húmicos; esto indica que M2 tiene un mayor contenido de minerales, reflejado en la conductividad ya que presentó un valor superior $(1990 \mu \mathrm{S} / \mathrm{cm})$ respecto a M1 $(3,01 \mu \mathrm{S} / \mathrm{cm})$.

\section{Caracterización microbiológica}

Los resultados microbiológicos se muestran en la tabla 3. Se encontró presencia de microorganismos mesófilos aerobios y anaerobios; este tipo de flora es normal en procesos de transformación, debido a la carga residente en el fruto y maquinaria utilizada en el proceso de pelado. Cabe mencionar que en el proceso de transformación no hay normatividad en cuanto a la carga microbiana debido a que el producto a consumir tiene una humedad del $9-12 \%$ por lo que la susceptibilidad a deterioro microbiano es baja, adicionalmente, para el consumo el grano pasa por un proceso de torrefacción en el que se realiza un proceso térmico disminuyendo la carga restante. En cuanto a coliformes y estafilococos la muestra M2 dió positivo, esto se puede deber, a la calidad de agua utilizada para el lavado.

Tabla 3. Resultados microbiológicos de las aguas residuales del café.

\begin{tabular}{ccc}
\hline \multicolumn{3}{c}{ Pruebas microbiológicas } \\
\hline Microorganismos & M1 & M2 \\
\hline M. aerobios (UFC/mL) & 3,74 & 4,56 \\
M. anaerobios (UFC/mL) & 5,79 & 5,24 \\
Estafilococos & Negativo & Positivo \\
Coliformes totales & Negativo & Positivo \\
\hline
\end{tabular}

La Organización Mundial de la Salud (OMS) sugiere que en el agua de consumo exista una cantidad de cero colonias de coliformes por $100 \mathrm{~mL}$ de agua [35], por lo que M2 no debe ser empleada para otros usos.

Adicionalmente, se resalta que las dos muestras de agua residual deben ser sometidas a procesos de tratamiento previo a su disposición en cuerpos de agua o directamente sobre el suelo, debido a que pueden afectar las especies animales y vegetales existentes en cuerpos hídricos y/o afectar procesos productivos, como es el caso de germinación de semillas, crecimiento de plantas, entre otras. Algunas alternativas que se emplean actualmente para este fin son: coagulación con extractos provenientes de semillas de Moringa oleífera [36], coagulación - floculación química y procesos de oxidación [37], oxidación enzimática [17], biometanización [38, 39], aireación y filtración con especies vegetales [40,41], entre otros.

Es importante definir la viabilidad técnica y económica de estas alternativas de acuerdo con las características de las zonas productivas y los volúmenes de aguas mieles producidos, así como nuevas alternativas de aprovechamiento para este 
residuo agroindustrial, en el marco del concepto de biorrefinería.

\section{Conclusión}

Las aguas residuales evaluadas tienen una composición química compleja que evidencia la necesidad de implementación de sistemas de tratamiento - aprovechamiento de estas. El tiempo de fermentación cumple un papel importante en la composición química de este subproducto generado en el procesamiento primario del café. Alternativas como la valorización de estos residuos con técnicas de extracción líquido-líquido podría permitir el pre-tratamiento y valorización en simultáneo de este importante residuo en la producción de café.

\section{Referencias bibliográficas}

[1] Valenzuela A. El café y sus efectos en la salud cardiovascular y en la salud materna. Rev Chil Nutr. 2010;37(4):514-23.

[2] United States Department of Agriculture (USDA). Coffee: World Markets and Trade. United States: Foreign Agricultural Service; 2018.

[3] Federación Nacional de Cafeteros de Colombia (FEDECAFE). La Política Cafetera 2010-2014. Economía Cafetera. Colombia: Federación Nacional de Cafeteros de Colombia; 2014.

[4] Banco Mundial. Estudio del sector cafetero en Colombia. Colombia: Federación de Cafeteros; 2018.

[5] Rodríguez N, Sanz JR, Oliveros CE, Ramírez CA. Manejo y disposición de los subproductos y de las aguas residuales del beneficio del café. Manual del cafetero colombiano: Investigación y tecnología para la sostenibilidad de la caficultura. Cenicafé. Colombia: Legis; 2013.

[6] Bermúdez Savón RC, García Oduardo N, Mourlot López A. Fermentación sólida para la producción de pleurotus $s p$. Sobre mezclas de pulpa de café y viruta de cedro. Tecnología Química. 2007;27(2):55-62.

[7] Iriondo-DeHond A, Garcia NA, Fernandez Gomez B, Guisantes Batan E, Velazquez Escobar $\mathrm{F}$, Blanch $\mathrm{P}$, et al. Validation of coffee by-products as novel food ingredients. Innovative Food Sci. Emerging Technol. 2019;51:194-204.

[8] Bertrand B, Rapidel B. Desafíos de la caficultura en Centroamérica. Costa Rica: Agroamérica;
1999.

[9] Tharian JA, Padmapriya R, Thirunalasundari T. Coffee waste management-An overview. Int. J. Curr. Sci. 2013;9:83-91.

[10] Aguilera Y, Consuegra R, Rapado M. Treatment of coffee wastewater by gamma radiation. In: International Atomic Energy Agency (IAEA), editors. Symposium on radiation technology for conservation of the environment; 1997 Sep 8-12; Zakopane, Poland. Vienna: International Atomic Energy Agency (IAEA); 1998. p. 21720.

[11] Pastrana L. Fundamentos de la fermentación en estado sólido y aplicación a la industria alimentaria. Cienc. Tecnol. Aliment. 1996;1(3):4-12

[12] Janissen B, Huynh T. Chemical composition and value-adding applications of coffee industry byproducts: A review. Resour., Conserv. Recycl. 2018;128:110-7.

[13] Association of Official Analytical Chemists. Official methods of analysis (fifteenth edition ed.). United States: Association of Official Analytical Chemists; 1990.

[14] Franson MAH. Métodos normalizados para el análisis de aguas potables y residuales. España: Ediciones Díaz de Santos; 1992.

[15] DadiD, Mengistie E, Terefe G, Getahun T, Haddis A, Birke W, et al. Assessment of the effluent quality of wet coffee processing wastewater and its influence on downstream water quality. Ecohydrol Hydrobiol. 2018;18(2):201-11.

[16] Aguiar LL, Andrade-Vieira LF, de Oliveira David JA. Evaluation of the toxic potential of coffee wastewater on seeds, roots and meristematic cells of Lactuca sativa L. Ecotoxicol Environ Saf. 2016;133:366-72.

[17] Chagas PMB, Torres JA, Silva MC, Corrêa AD. Immobilized soybean hull peroxidase for the oxidation of phenolic compounds in coffee processing wastewater. Int J Biol Macromol. 2015;81:568-75.

[18] Novita E. Biodegradability Simulation of Coffee Wastewater Using Instant Coffee. Agric Agric Sci Procedia. 2016;9:217-29.

[19] Rattan S, Parande AK, Nagaraju VD, Ghiwari GK. A comprehensive review on utilization of wastewater from coffee processing. Environ Sci Pollut Res. 2015;22(9):6461-72.

[20] Córdoba NM, Guerrero JE. Caracterización de los procesos tradicionales de fermentación de café en el departamento de Nariño. Biotecnol. Sect. Agropecu. Agroind. 2016;14:75-83. 
[21] Ministerio de Ambiente y Desarrollo Sostenible. Resolución 631 de 2015 - Parámetros vertimientos. Colombia; 2015.

[22] Orozco C, Barrientos $\mathrm{H}$, Lopezlena A, Cruz J, Selvas C, Osorio E, et al. Evaluación de una planta piloto de aguas residuales del café. Higiene y Sanidad Ambiental. 2005;5:123-31.

[23] Fia R, De Matos AT, Lambert TF, Fia FRL, De Matos MP. Tratamento das águas do processamento dos frutos do cafeeiro em filtro anaeróbio seguido por sistema alagado construído: li - remoção de nutrientes e compostos fenólicos. Eng. Agric. 2010;30(6):1203-13

[24] Alvarez J, Hugh S, Cuba N, Loza-Murguia M. Evaluación de un sistema de tratamiento de aguas residuales del prebeneficiado de café (Coffea arabica) implementado en la comunidad Carmen Pampa provincia Nor Yungas del Departamento de La Paz. Journal of the Selva Andina Research Society. 2011;2(1):34-42

[25] Rodríguez S, Pérez RM, Fernández M. Estudio de la biodegradabilidad anaerobia de las aguas residuales del beneficio húmedo del café. Interciencia. 2000;25(8):386-90.

[26] Del Real Olvera J, Gutiérrez JI. Biodegradación anaerobia de las aguas generadas en el despulpado del café. Rev. Colomb. Biotecnol. 2010;12(2):230-39.

[27] Vásquez N, Rodríguez J, Torres P, Madera C. Desempeño del proceso de estabilización por contacto para el tratamiento del agua residual doméstica de Cali, Colombia. DYNA. 2011;78(168):98-107.

[28] Zambrano DA, Isaza JD, Rodríguez N, López $\mathrm{U}$. Tratamiento de aguas residuales del lavado del café. Cenicafé. 1999;20:1-30.

[29] Crombet S, Abalos A, Rodríguez S, Pérez N. Evaluación del tratamiento anaerobio de las aguas residuales de una comunidad universitaria. Rev. Colomb. Biotecnol. 2016;18:49-56.

[30] Molina AE, Villatoro RA. Propuesta de tratamientos de aguas residuales en beneficios húmedos de café (trabajo de grado). San Salvador, El Salvador: Universidad de El Salvador; 2006.

[31]Lin K, Pei J, Li P, Ma J, Li Q, Yuan D. Simultaneous determination of total dissolved nitrogen and total dissolved phosphorus in natural waters with an on-line UV and thermal digestion. Talanta. 2018;185:419-26

[32] Cárdenas GL, Sánchez IA. Nitrógeno en aguas residuales: orígenes, efectos y mecanismos de remoción para preservar el ambiente y la salud pública. Universidad y Salud. 2013;15:72-88.

[33] Universidad Nacional Autónoma de México. Tratamiento de aguas. Manual de laboratorio. México: Universidad Nacional Autónoma de México; 2013.

[34] Dadi D, Mengistie E, Terefe G, et al. Assessment of the effluent quality of wet coffee processing wastewater and its influence on downstream water quality. Ecohydrology \& Hydrobiology. 2018;18(2):201-11.

[35] Organización Mundial de la Salud (OMS). Guías para la calidad del agua potable. Vol. 1. 3 ed. Suiza: Organización Mundial de la Salud (OMS); 2006.

[36] Garde WK, Buchberger SG, Wendell D, Kupferle MJ. Application of Moringa Oleifera seed extract to treat coffee fermentation wastewater. Journal of Hazardous Materials. 2017;329:102-9.

[37]Zayas Péerez T, Geissler G, Hernandez F. Chemical oxygen demand reduction in coffee wastewater through chemical flocculation and advanced oxidation processes. J Environ Sci. 2007;19:300-5.

[38] Botello Suárez WA, da Silva Vantini J, Duda $\mathrm{RM}$, et al. Predominance of syntrophic bacteria, Methanosaeta and Methanoculleus in a twostage up-flow anaerobic sludge blanket reactor treating coffee processing wastewater at high organic loading rate. Bioresour. Technol. 2018;268:158-68.

[39] Selvamurugan $M$, Doraisamy $P$, Maheswari M. An integrated treatment system for coffee processing wastewater using anaerobic and aerobic process. Ecological Engineering. 2010;36(12):1686-90.

[40] Rossmann M, De Matos AT, Abreu EC, E Silva FF, Borges AC. Performance of constructed wetlands in the treatment of aerated coffee processing wastewater: Removal of nutrients and phenolic compounds. Ecological Engineering. 2012;49:264-9.

[41]Rossmann M, De Matos AT, Abreu EC, E Silva FF, Borges AC. Effect of influent aeration on removal of organic matter from coffee processing wastewater in constructed wetlands. J. Environ. Manage. 2013;128:912-9. 